\title{
RELACIÓN AFECTIVA DE MUJ ERES CON UN ESPOSO ALCOHÓLICO: UN COMPORTAMIENTO SOCIAL APRENDIDO QUE REPERCUTE EN SU SALUD
}

Relação afetiva das mulheres com um cônjuge alcoólico: um comportamento social aprendido que repercute em sua saúde

Affective relationship between women with an alcoholic husband: a learned social behavior that affects your health

Maria Alejandra Hernández Castañón ${ }^{1}$

Margarita Antonia Villar Luis²

\section{RESUMEN}

Se realiza una revisión sobre el tema de la codependencia y su relación con comportamientos socialmente aprendidos sobre la base de una división de género. La codependencia en mujeres de adictos a alcohol y sus consecuencias individuales, familiares y comunitarias también se mencionan. En este contexto se realiza una breve síntesis de los patrones de comportamiento derivados del rol social asignado a la mujer en México en relación con esta temática. Finalmente se establecen algunas posturas teóricas para el análisis de la codependencia a partir del análisis socio-histórico de los significados que tiene para la población el consumo abusivo de alcohol y la relación patológica establecida que establece la mujer con el marido. En este sentido, diferentes autores mencionan la importancia de considerar a la familia y no sólo al individuo para la atención, ya que las consecuencias físicas y mentales están presentes tanto en hombres como mujeres.

Palabras clave: Mujeres. Alcoholismo. Codependencia. Identidad de género. Salud.

\section{Resumo}

Trata-se de uma revisão sobre o tema codependência e sua relação com comportamentos socialmente aprendidos fundamentada na divisão de gênero. A codependência em mulheres com companheiros dependentes de álcool e suas conseqüências para o indivíduo, família e comunidade também são abordadas. Nesse contexto, realiza-se uma breve síntese dos padrões de comportamento derivados do papel social atribuído à mulher no México em relação ao tema. Por fim, são estabelecidos alguns posicionamentos teóricos na análise da codependência a partir da análise sócio-histórica dos significados que a população atribui ao consumo abusivo de álcool e da relação patológica que a mulher estabelece com seu marido. Diferentes autores referem a importância de considerar a família, e não somente o usuário, no seu cuidado, já que as conseqüências físicas e mentais estão presentes tanto no homem quanto na mulher.

Palavras-chave: Mulheres. Alcoolismo. Codependência. Identidade de Gênero. Saúde.

\begin{abstract}
This is a review about codependence and its relation with socially learned behaviors based on a gender division. Codependence in women of alcohol addicts and its individual, family and Community consequences are also mentioned. In this context, a short synthesis is presented of the behavioral standards derived from the social role assigned to women in Mexico with respect to this theme. Finally, some theoretical positions are established to analyze codependence based on the sociohistorical analysis of the meanings the population attributes to alcohol abuse and the pathological relation women establish with their husband. In this sense, different authors mention the importance of considering the family and not only the individual for care, as the physical and mental consequences are present in women as well as men.
\end{abstract}

Keywords: Women. Alcoholism. Codependence. Gender identity. Health.

\footnotetext{
'Profesor-Doctor de la Facultad de Enfermería - Universidad Autónoma de Querétaro/México e-mail: alehdez983@yahoo.com.mx 2 Profesor Doctor. Coordinador del Proyecto CICAD/OEA \& EERP.USP. Titular del Departamento de Enfermería Psiquiátrica y Ciencias Humanas. Escuela de Enfermería de Ribeirão Preto. Centro colaborador de la OMS para el Desarrollo de la Investigación en Enfermería. e-mail: margarit@eerp.usp.br
} 


\section{INTRODUCCIÓN}

En el año 2000, el consumo de alcohol fue responsable de $4 \%$ de la carga mundial de morbilidad. En países desarrollados se atribuye al alcohol el $9.2 \%$ de los años de vida ajustados en función de la discapacidad (AVAD), resultado de trastornos neuropsiquiátricos y de traumatismos no intencionales y del $6.2 \%$ de los AVAD perdidos ${ }^{1}$

En los países de América Latina y el Caribe (ALC), las diferencias de género son agudas, pues son los hombres quienes representan mayores problemas relacionados con el alcohol, con base a normas existentes que apoyan y estimulan el beber entre los hombres, el género no está explícitamente considerado en las intervenciones actuales. Con excepción de México, Brasil, Chile y Costa Rica, los países de ALC no han mostrado un interés sostenido en la conducción de estudios sobre el consumo de alcohol ${ }^{2}$.

Sin embargo, el desarrollo del conocimiento sobre alcoholismo como enfermedad ha enfatizado sus aspectos negativos, por lo que las investigaciones se han centrado en la embriaguez como el desvío individual y no como un comportamiento social ${ }^{3}$, pues el problema del alcohol no afecta únicamente a quien tiene la adicción, sino que involucra a la familia, especialmente a las mujeres, quienes cuidan y se responsabilizan del alcohólico en el diario convivir.

Se refiere que siempre que uno de los miembros de la familia desarrolla una conducta adictiva, otro desarrolla codependencia 4 . En este contexto, el uso y abuso de sustancias adictivas como el alcohol y las drogas va en incremento, así como situaciones de violencia físico, sexual y psicológica, que resultan de la relación adicto/codependiente, dañando la autoestima de los integrantes de la familia, en especial a las mujeres.

De ahí que el objetivo de la revisión fue: describir algunas posturas teóricas sobre el tema codependencia, especialmente en mujeres con cónyuges alcohólicos, como parte de un estudio sobre su mundo de la vida.

\section{METODOLOGÍA}

Se realizó una revisión bibliográfica con base en los descriptores de codependencia, alcoholismo, mujer, género y salud, para lo cual se consideraron artículos de 1990 a la fecha. De igual forma, se identificaron algunos estudios representativos para el contexto mexicano realizados en la década de los 90, que hacen un análisis histórico del tema. de su posición social. La búsqueda en Internet se realizó durante los meses de junio y julio de 2004 y actualizándose información para los meses de agosto-septiembre 2007, tomando como referencia las palabras claves antes mencionadas, utilizando base de datos scielo y buscador google académico, por las características cualitativas y más del ámbito social que se procuraba. También se realizó consulta bibliografía impresa específica y discusión con especialistas en el tema, al participar en eventos científicos relacionados con la salud de la mujer y género. Con esta información, se elaboró la propuesta que se presenta a seguir.

\section{RESULTADOS}

La codependenciase expresa como la aceptación del engaño y maltrato de que son objeto las mujeres por parte de quien consume algún tipo de droga, perdiendo su equilibrio emocional, alterando su forma de pensar, sentir y su comportamiento. Si la persona dependiente es la pareja, la relación afectiva y sexual se deteriora así como su vida cotidiana y salud ${ }^{5}$, hasta el punto de vivir por y para él ${ }^{6}$.

Los comportamientos característicos de la codependencia son: a) conductas determinadas por fuerzas externas y no por decisiones voluntarias; $b$ ) inadecuado manejo de sentimientos, equiparando amar con sufrir y sacrificarse, c) protección y disculpa al adicto; c) baja autoestima; d) comportamientos de control hacia otras personas y eventos, e) estrés, enfermedades psicosomáticas y depresión?

La codependencia se puede adquirir de forma inducida directa mediante un sistema de conductas y creencias familiares que no permiten la expresión de emociones y afectos; de manera incidental al momento de la unión con otra persona, reaprendiendo formas disfuncionales de expresar y sentir afecto; e inducida por normas institucionales que dificultan al codependiente negar ayuda a otra persona, aún en perjuicio propio, generando sentimientos de angustia y baja autoestima ${ }^{8}$.

Uno de los grupos más propensos a la codependencia son los cónyuges de alcohólicos 0 adictos, sus comportamientos son de víctima y mártir, de perseguidora y salvadora, pues considera que solo ella puede salvar y brindar la ayuda al adicto, descalificando a terapeutas o centros de tratamiento $0^{4}$, de ahí que se considere a la codependencia como una patología propia de la mujer, con alta prevalencia determinada por aspectos sociales y culturales ${ }^{6}$

Por lo expresado anteriormente, se observa una fuerte relación entre codependiencia y género. El sistema de género legitima y reproduce las prácticas, representaciones, normas y valores que la sociedad construye a partir de la diferencia sexual ${ }^{9}$.

El sistema sexo/género se definió como un conjunto de arreglos a través de los cuales una sociedad trasforma la sexualidad biológica en productos de la actividad humana, en las que estas necesidades sociales trasformadas son satisfechas. En la década de los 70's, se hacía una diferenciación entre los términos sexo y género, el primero indicaba las diferencias biológicas entre un hombre y mujer; el segundo, se refería a las construcciones sociales, culturales, psicológicas que se imponen sobre esa diferencia biológica ${ }^{10}$.

Sin embargo, las propuestas teóricas relativas al género aparecen al final del siglo XX como construcción social, donde el sentido de las actividades se adquiere por medio de interrelaciones sociales en un contexto de desigualdad. El 
concepto de género implica los símbolos culturalmente disponibles, los conceptos normativos que sustentan las interpretaciones del sentido de los símbolos, las referencia institucionales y la identidad subjetiva, ésta última construida en relación a una serie de actividades, organizaciones sociales y representaciones culturales históricamente situadas ${ }^{11}$.

Las normas sociales en torno a la posición de la mujer dentro de la sociedad mexicana se sustentan en ese reconocimiento. Un estudio describe el comportamiento esperado de la mujer entre el periodo de la colonia y primera mitad del siglo XX que se resume en: obediencia total al cónyuge a cambio de sostén y protección, sobriedad sexual, recato, honestidad y mesura, donde el castigo físico se asociaba a la purificación, posiblemente interiorizado por el hombre para ejercerlo y por la mujer para soportarlo, pues su papel de cohesionadora del núcleo familiar, socializadora y reproductora de los valores dentro del hogar, ha soslayado la violencia familiar ${ }^{12}$.

La familia se convier te en la reproductora principal de la ideología y construcción del género con todas sus implicaciones, entre ellas las relaciones de dominación que se ejerce sobre la mujer. Se menciona que los individuos aprenden un patrón de comportamiento, interiorizando las sanciones que corresponden a la violación de un imperativo generalizado y con ello incorporando el poder del grupo social ${ }^{13}$.

En este contexto, las tradiciones y creencias emanadas del sistema social organizado a partir de una premisa de género, vinculan a la mujer con el hogar, de ahí que, la dinámica de las familias de adictos parta de un proceso cíclico que involucra a tres personas o más, generalmente el adicto y los padres. En esta dinámica, el tema de la droga no se discute e inicialmente no se da importancia a la adicción, después tienen esperanza de su cura y finalmente, niegan la enfermedad y rechazan al adicto. La disfunción se convier te en estilo de vida familiar con reglas confusas, especialmente para los niños, que puede facilitar el desarrollo de adicciones o de relaciones enfermas a futuro ${ }^{14}$.

Se menciona que las instituciones sociales retoman criterios de validez, que provienen del reconocimiento intersubjetivo, por lo que los individuos asumen actitudes y roles que los demás miembros esperan de ellos ${ }^{13}$, de ahí que el concepto de género se aplica a las relaciones de desigualdad entre mujeres y hombres en torno a la distribución de los recursos, responsabilidades y poder.

En lo relativo a la salud, también se observa esta diferencia cuando se establece una mayor mortalidad masculina, 5 a 20 veces más que en las mujeres en población adulta joven, esto debido a comportamientos de riesgo que derivan en accidentes, violencias y suicidios, asociados con la división social de roles según sexo y las correspondientes expectativas culturales de hombría que son tolerados y estimulados entre los varones ${ }^{15}$, como lo es el alcoholismo.

\section{DISCUSIÓN}

De acuerdo con las revisión realizada, el comportamiento de la mujer mexicana no se ha modificado de manera sustancial, es decir, prevalece el concepto de subordinación y sacrificio ${ }^{16}$. Estos antecedentes, en los cuales las creencias, tradiciones y apariencias sociales son muy importantes, los problemas se mantienen al interior del seno familiar, de ahí que cuando se presenta dependencia a sustancias adictivas en un miembro de la familia, esto no se comente con nadie ajeno y mucho menos se solicite ayuda profesional, como se muestra en algunos estudios ${ }^{12,14,17}$

El reconocimiento de esta problemática es fundamental al momento de estudiar el comportamiento de la mujer que convive con una persona alcohólica, especialmente aquella que tiene un compromiso de unión, puesto que en el proceso de socialización, el patrón generalizado de comportamiento cobra para el individuo la autoridad de un debes y con ello, el tipo de validez normativa en virtud del cual tienen las normas fuerza vinculante $^{13,}$, es decir, en el caso de las adicciones, los comportamientos esperados en ellas son el cuidado del otro, pero paradójicamente, también depender del marido.

Las mujeres procuran los servicios de salud en busca de orientación, cuando su situación de convivencia con el alcohólico llegaba a un punto límite como pérdida de empleo, pero sobre todo por complicaciones clínicas graves como cirrosis hepática y complicaciones cardiovasculares, resultado del abuso en su forma de beber ${ }^{15}$. Ellas desarrollan un sistema de defensa eficaz más no productivo, pues se encuentran llenas de rabia, preconceptos, indiferencias y frustración con la relación, contrastando con la responsabilidad y afecto que sentían por el alcohólico ${ }^{17}$.

Diferentes estudios sobre codependencia, muestran en esta relación la presencia de violencia intrafamiliar, sin embargo, centrar el problema en la conducta individual del hombre y no como resultado de un desequilibrio de poder entre los individuos, da una falsa visión del fenómen $0^{18}$, ya que el ejercicio de poder -inter e intragéneros-, tiene efectos claramente negativos sobre la integridad física, psicológica y social no solo de las mujeres, sino también de los hombres ${ }^{15}$.

Con base a lo anterior, los profesionales de la salud requieren una formación que les permita tener una visión integral de los problemas de salud, ya que como en el caso del alcoholismo, no solo se trata del dependiente, sino del codependiente y de la propia familia, de ahí que las instituciones educativas deben implementar programas educativos con contenidos sobre salud mental, aspectos socio-antropológicos y psicológicos, que proporcionen a los estudiantes herramientas para identificar y hacer frente a este tipo de problemas, ya que muchas veces la falta de capacitación, puede ser una barrera para reconocerlos y brindar atención oportuna.

En el caso específico de Enfermería, la adquisición de conocimiento científico-técnicos, que le permitan tener una 
visión general de los problemas biológicos, psicológicos y sociales que conforman el fenómeno de la dependencia al alcohol, pero sobre todo, capitalizar el reconocimiento social que la profesión posee en actividades de salud comunitaria ${ }^{17}$.

Esto le permitirá proponer y realizar actividades conjuntas con la comunidad, tendientes a la prevención y reducción del consumo de alcohol, además de coordinarse con otros profesionales, con la finalidad de buscar modificar el habito de beber de dependiente, pero sobre todo, que influir en la modificación de patrones de comportamiento ${ }^{13}$ que sustentan la codependencia.

\section{CONCLUSIONES}

El alcoholismo y la codependencia son la misma enfermedad, como cualquier adicción comparten características de negación, obsesión, compulsión y pérdida de control, ambos son comportamientos socialmente aprendidos que afectan la salud física y mental tanto de quien consume, como de aquellos que conviven cotidianamente con el adicto.

Es posible que en este comportamiento aprendido por nuestras madres y abuelas, todavía exista un fuerte remanente, que condicione el actuar de la mujer ante una problemática familiar como es el uso y abuso del alcohol.

De ahí que en la revisión realizada, se observó que el uso y abuso de sustancias adictivas va en incremento, así como el abuso físico, sexual y psicológico, resultante de la relación disfuncional adicto/codependiente, dañando la autoestima de los integrantes del núcleo familiar, especialmente en mujeres, es decir, hay cambios significativos en el mundo de la vida de los directamente involucrados con el fenómeno de la codependencia ${ }^{13}$.

Se establece que el rol social asumido por los individuos, es determinante en el tipo de acción que lleven a cabo, con base a normas legítimamente reguladas, a par tir de las cuales, los miembros de una comunidad desarrollan una identidad social que sentará las bases para la conformación de su mundo subjetivo.

Por lo que la relación disfuncional que se observa en la familia, puede ser el reflejo de lo que acontece en su contexto social, donde los medios de comunicación han dado un nuevo sentido al consumo de alcohol, relacionándolo con una imagen de éxito; la pérdida de control social manifestado por la trasgresión de normas, como son el consumo en la vía pública y la venta a menores de edad cuando está prohibido por ley; así como el incremento de patologías relacionadas al consumo abusivo de alcohol.

De ahí que dimensionar el alcoholismo, ya no sólo como un problema individual, sino un fenómeno multi-causal, que desde la perspectiva de Habermas, tendría que evaluar los distintos procesos de la reproducción, es decir, la racionalidad del saber, la solidaridad de los miembros y la capacidad personal para responder autónomamente de sus acciones ${ }^{13}$.
Cuando la actitud de la mujer cambie no de manera aislada, sino como grupo social, es decir, cuente con redes sociales, familiares e institucionales que les permitan modificar ideas y creencias en torno al alcohol y al deber ser de la mujer, sólo entonces será posible que tomen una postura específica y sus acciones sean asertivas para enfrentar el fenómeno y mejorar su calidad de vida individual y familiar.

Es importante estar concientes de la complejidad del proceso involucrado en la constitución de actitudes, para que enfermería y otros miembros del equipo de salud, no tengan expectativas de cambios a corto plazo en las mujeres de alcohólicos, porque así como llevó tiempo para construir esa identidad y modos de interactuar, la desconstrucción y reconstrucción, es un proceso que también requiere tiempo y apoyo profesional.

\section{Referências}

1.Organización Mundial de la Salud-OMS. Problemas de salud pública causados por el uso nocivo del alcohol. $58^{\mathrm{a}}$ Asamblea Mundial de la Salud 2005. [citado 20 ago 2007]; 1-4. Disponible en: http:// www.who.int/substance_abuse

2.Pyne H, Cleason M, Correia M. Dimensiones de género sobre el consumo de alcohol y problemas afines en América Latina y el Caribe. Banco Mundial 2002 [citado 24 ene 2007]; [3-12]. Disponible en: http://Inweb18.worldbank.org

3.Pessanha D. Alcoolismo: acusação ou diagnóstico? Cad Saude Publica 2004 jan/fev; 20(1): 7-13.

4.Guevara W. La codependencia, una forma de convivir con el sufrimiento. Boletín Consejo Superior de Investigaciones CSI/UNMSM [on-line] 2003 [citado 29 oct 2007]; (48): 13-15. Disponible en: http://sisbib.unmsm.edu.pe

5.Red Interamericana para la Prevención de Drogas- RIPRED. Guía para la mujer sobre las drogas. [citado 13 jun 2004]. [aprox. 34 telas]. Disponible en: http://www.dpna.org

6.Mansilla F. Codependencia y psicoterapia interpersonal. Rev Asoc Esp Neuropsiq 2001; 23 (81): 9-35.

7.Pérez A, Delgado D. La codependencia en familias de consumidores y no consumidores de drogas: estado del arte y construcción de un instrumento. Psicothema. [on-line]. Universidad de Oviedo, 2003; [citado 15 sept 2007]; 11(4): 381-387. Disponible en: http:// redalyc.uaemex.mx

8.Vacca R. La co-adicción, aspectos culturales y clínicos para su aprendizaje. Rev Peruana Drogodependencias [on-line] 2003; [citado 30 oct 2007] 1 (1):231-253. Disponible en: http://www.devida.gob.pe

9.Beatres G. La perspectiva de género como modelo de análisis de la violencia familiar y el consumo de alcohol y otras drogas. Reunión del Grupo de Consulta sobre el Impacto del Abuso de Drogas en la Mujer y la Familia. Secretaría Ejecutiva de la Comisión Interamericana para el Control y Abuso de Drogas- CICAD/OEA Montevideo (UR); 1996. p. 146.

10.Mezan L, Piscitelli A, Goldani A. A prática feminista e o conceito de gênero. Campinas(SP): IFCH/UNICAMP; 2002. 
11.Scott J. Gênero: uma categoría útil para análise histórica. Tradução de Christine Rufino Dabat, María Betânia Avila. Recife (PE): SOS CORPO; 1991.

12.Suárez M. Discurso, género y violencia intrafamiliar en la historia moderna de México. ¿Una memoría. Archivo Tiempo y Escritura 2004. [citado 24 ene 2007]. Disponible en: http://www.azc.unam.mx

13. Habermas J. Teoría de la acción comunicativa, 2. Crítica de la razón funcionalista. $1^{\mathrm{a}}$ ed. México(MX): Taurus; 2002.

14.Coddou A, Chadwick M. Evolución del concepto de codependencia. Chile Med 1999. [citado 20 jun 2004]; [aprox. 18 telas]. Disponible en: http://www.eradicciones.org

15.Gómez Gómez E. Equidad, género y salud: retos para la acción. Rev Panam Salud Publica [on-line] 2002 may/jun; [citado 16 mar 2007]; 11(5/6): 454-61. Disponible en: http://www.scielosp.org.
16.Diaz-Guerrero R. Bajo las garras de la cultura. Psicología del mexicano. México(MX): Trillas; 2003.

17.Almeida TR, Villar-Luis MA. 0 cuidar de quem cuida: uma visão sobre os cuidadores de alcoolistas. In: Villar Luis M, Pillon, C. Assistência a usuários de álcool e drogas no Estado de São Paulo: uma amostra de servicios e programas. Ribeirão Preto (SP): FIERP/ USP; 2004.

18.Castro R, Riquer F. La investigación sobre violencia contra las mujeres en América Latina: entre el empirismo ciego y la teoría sin datos. Cad Saude Publica [on-line] 2003 jan/fev. [citado 10 out 2007]; 19 (1): 135-46. Disponible en: http://www.scielosp.org. 